\title{
Calculation of equivalent magnetic permeability of ferromagnetic materials for modeling of three-dimensional eddy current fields
}

\author{
Sergey Karpushkin ${ }^{1, *}$, Aleksey Glebov ${ }^{1}$, and Sergey Karpov ${ }^{1}$ \\ ${ }^{1}$ Tambov State Technical University, 392000, Sovetskaya, 106, Tambov, Russia
}

\begin{abstract}
The paper presents a method of calculating a constant value of magnetic permeability of ferromagnetic materials, equivalent to the basic magnetization curve by the criterion of real power. The proposed method makes it possible to use linear differential equations of the electromagnetic field in the analysis of three-dimensional fields of eddy currents in ferromagnetic materials, thus significantly reducing the calculation time. We propose a method of calculating the power of inductors using two-dimensional axisymmetric models. The scope of the proposed methods is limited to the case of placement of the inductor inside the ferromagnetic body.
\end{abstract}

\section{Introduction}

The problem of calculating eddy current fields is of great practical importance for the analysis of electrical devices for different purposes. A special role in this equipment segment is given to induction heating devices, the functioning of which largely depends on the configuration of the electromagnetic field.

Eddy currents are induced most effectively in ferromagnetic materials, which are characterized by a non-linear relationship between induction and magnetic field strength. This dependence is expressed as the magnetization curve, and the stage of mathematical modeling it results in the formation of a system of nonlinear time-dependent vector differential equations in partial derivatives. The solution of such a system in a three-dimensional representation is quite complicated.

Assuming the linearity of magnetic properties of the material, the electromagnetic field equations are considerably simplified. The use of complex numbers makes it possible to get rid of differentiation with respect to time, and the model equations take a quasi-stationary form.

However, ferromagnetic materials with linear magnetic properties are not found in practice. In this regard, it is relevant to develop methods of calculating constant values of magnetic permeability, ensuring that the heating capacities in linear and non-linear calculations are the same. In other words, there is a need to replace the original magnetization curve with the equivalent value of magnetic permeability.

\section{Method for calculating equivalent magnetic permeability}

The electromagnetic field equations when using the concept of magnetic vector potential $\mathbf{A}(\mathrm{Wb} / \mathrm{m})$ and electric scalar potential $V(\mathrm{~W})$ are of the form [1]:

$$
\begin{gathered}
\operatorname{rot}\left(\mu^{-1} \operatorname{rot} \mathbf{A}\right)-\operatorname{grad}\left(\mu^{-1} \operatorname{div} \mathbf{A}\right)+\gamma \frac{\partial \mathbf{A}}{\partial t}+\gamma \operatorname{grad} V=0 ; \\
\operatorname{div}\left(-\gamma \frac{\partial \mathbf{A}}{\partial t}-\gamma \operatorname{grad} V\right)=0,
\end{gathered}
$$

where $\mu$ is absolute magnetic permeability, $\mathrm{H} / \mathrm{m} ; \gamma$ is specific electric conductivity, $\mathrm{ohm}^{-1} \mathrm{~m}^{-1} ; t$ is time, $\mathrm{s}$.

Equations (1)-(2) are written for an isotropic material in the existence of eddy currents and derived under assumptions about the absence of Maxwell's displacement current, as well as the absence of the phenomenon of magnetic hysteresis. Typically, in induction heating these phenomena can be disregarded.

To solve equations (1)-(2) a method of finite elements realized in various CAE-systems is the most effective. The authors of this study used ANSYS system of finite-element analysis. Modeling of an electromagnetic field described by equations (1)-(2) was done using SOLID97 finite elements (Fig. 1). Finite elements of tetrahedral shape are more versatile to split solids of arbitrary form.

Modeling of induction heating involves a step-be step analysis, using: 1) calculation of eddy currents with the use of SOLID97 finite elements; 2) thermal analysis using SOLID70 finite elements, the geometry of which is similar to those shown in Fig. 1. Thus, for electromagnetic and thermal analyses a unified finite element mesh is used; changing only the type of the finite elements when conducting the thermal analysis $[2,3]$.

\footnotetext{
"Corresponding author: karp@mail.gaps.tstu.ru
} 

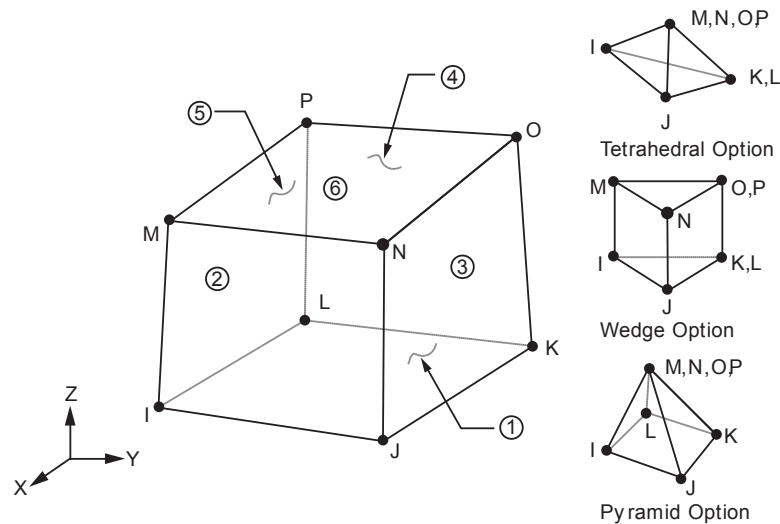

Fig. 1. Geometry of SOLID97 finite element of the ANSYS system

Solution of the three-dimensional nonlinear equations (1)-(2) using the finite element method (FEM) is costly in terms of computing time because of the low rate of convergence of iterations and time-dependent electromagnetic processes. At each time point in the nodes of the finite element mesh it is required to determine the four unknowns: electric scalar potential $V$ and the three components of the magnetic vector potential A. If magnetic permeability is constant, then for sinusoidal supply voltage expressions (1)-(2) are simplified to linear equations in a quasi-stationary complex representation:

$$
\begin{gathered}
-\mu^{-1} \Delta \dot{\mathbf{A}}+j 2 \pi f \gamma \dot{\mathbf{A}}+\gamma \operatorname{grad} \dot{V}=0 ; \\
\operatorname{div}(-j 2 \pi f \gamma \dot{\mathbf{A}}-\gamma \operatorname{grad} \dot{V})=0,
\end{gathered}
$$

where $j$ is an imaginary unit $\left(j^{2}=-1\right)$.

Time required to solve equations (3)-(4), as compared with that required to find solutions of the initial equations (1)-(2) can be reduced by more than one order of magnitude. However, the need for a constant value of magnetic permeability equivalent to the magnetization curve by average real power for one period prevents their direct application to calculate the eddy current fields in ferromagnetic materials.

This problem can be solved by a method based on the solution of two-dimensional nonlinear equations of electromagnetic field (in this case, the mathematical model is greatly simplified). Assuming perpendicularity of the external current to the $X Y$ plane, the magnetic vector potential has only one component $A_{z}$, and the scalar electric potential is not used [4]:

$$
\operatorname{rot}\left(\mu^{-1} \operatorname{rot} A_{z}\right)+\gamma \frac{\partial A_{z}}{\partial t}=0
$$

Thus, in every point of the calculation domain it is required to find only one unknown $A_{z}$ (in a threedimensional analysis the number of unknowns is four), i.e. it is not difficult to take into account nonlinear characteristics of ferromagnets in a two-dimensional problem. Two-dimensional models can be quite effectively in the cases, in which their application does not lead to distortion of the description of physical processes.

Linear equations (3)-(4) are simplified in a similar way in a two-dimensional formulation:

$$
-\mu^{-1} \Delta \dot{A}_{z}+j 2 \pi f \gamma \dot{A}_{z}=0 .
$$

The dependence of equivalent permeability on the characteristics of the inductor and its position was investigated by solving the equations (5) and (6) for the axisymmetric system, consisting of a ferromagnetic disc and an inductor (Fig. 2). The radius of the inductor location, the number of its winding turns, the current, the fill factor (the proportion of "pure" wire in the total volume of the slot), the position of the inductor relative to the outer surfaces of the disc ranged alternately. For each combination we conducted a "standard" non-linear calculation, followed by a series of linear calculations, in the course of which the equivalent value of magnetic permeability was determined.

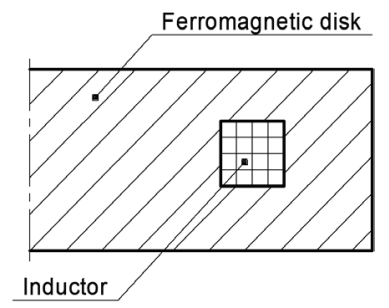

Fig. 2. Axisymmetric model with a disk and an inductor

The computational experiments resulted in the following findings:

- magnetic permeability is mostly affected by crosssectional dimensions of the inductor and its magnetomotive force (MMF);

- the impact of the inductor location radius can be ignored if it exceeds the width of the inductor more than twice;

- the value of the distance from the inductor to the outer surfaces of the disc substantially affects the eddy currents if this distance is less than the doubled depth of penetration of electromagnetic waves in the body of the disc;

- the fill factor of the inductor slot with wire affects only the inductor heat generation (resistive heating);

- the location of the inductor inside the slot and the gap between the inductor and the slot walls do not affect the induced eddy currents due to shielding.

The findings allowed offering a method of determining the equivalent permeability, shown in Fig. 3. The solutions cycle continues as long as the values of real power the inductor in linear and nonlinear calculations ( $P_{\text {lin }}$ и $P_{n l i n}$ respectively) differ by more than permissible error value $\varepsilon$. The obtained value of magnetic permeability is used in three-dimensional calculation to solve the system of equations (3)-(4). The real power is calculated by the Joule-Lenz law. For a nonstationary model described by the nonlinear equation (5) the calculation of the average power in one period is done by the formula: 


$$
P_{n l i n}=\frac{1}{T} \int_{t_{s t}}^{t_{s t}+T} \int_{\Omega} \frac{J_{z}^{2}}{\gamma} d \Omega d t
$$

where $T$ is the period of the current source fluctuations, s; $\Omega$ is the region of existence of eddy currents (the disk region); $J_{z}$ is a $\mathrm{Z}$-component of the density vector of eddy currents $J$ (in the two dimensional formulation the other components are equal to zero), $\mathrm{A} / \mathrm{m}^{2} ; t_{s t}$ is conditional stabilization time of electromagnetic processes, s; when it finishes, changes in amplitude values of the current density $J_{z}$ can be neglected.

Time $t_{s t}$ is determined at a stage of preliminary computational experiments and generally does not exceed $3 T$.

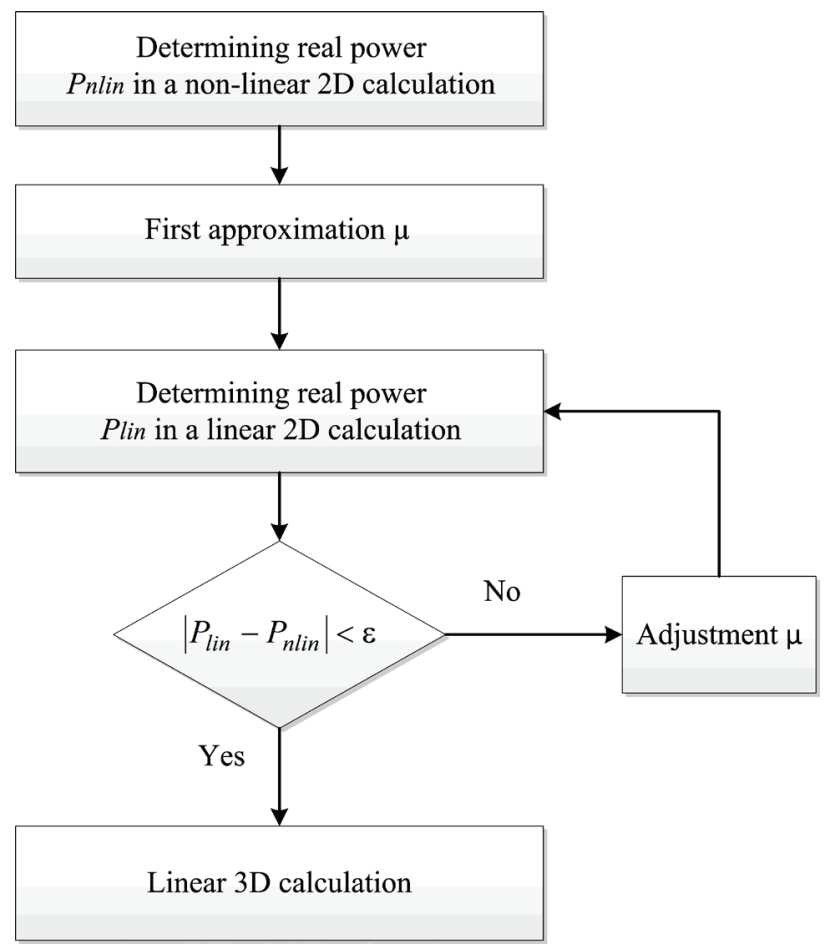

Fig. 3. A flowchart of determining the equivalent magnetic permeability

The eddy currents density in the two-dimensional formulation is calculated by the formula:

$$
J_{z}=-\gamma \frac{\partial A_{z}}{\partial t}
$$

When using the complex linear equation (6) the average power in one period will be

$$
P_{\text {lin }}=\int_{\Omega} \frac{\left|J_{z}\right|^{2}}{2 \gamma} d \Omega,
$$

where $\left|J_{z}\right|=\sqrt{\operatorname{Re} \dot{J}_{z}^{2}+\operatorname{Im} \dot{J}_{z}^{2}}$ is the module of complex current density $\dot{J}_{z}$.

By using this method we obtained and approximated by power function the dependences of equivalent magnetic permeability of ferromagnetic structural steel and the MMF of the inductor sized $25 \times 25 \mathrm{~mm}$ when connected to an ideal current generator (Fig. 4, curve 1) and an ideal voltage generator (Fig. 4 , curve 2). In the calculations, we used the magnetization curve described in [5].

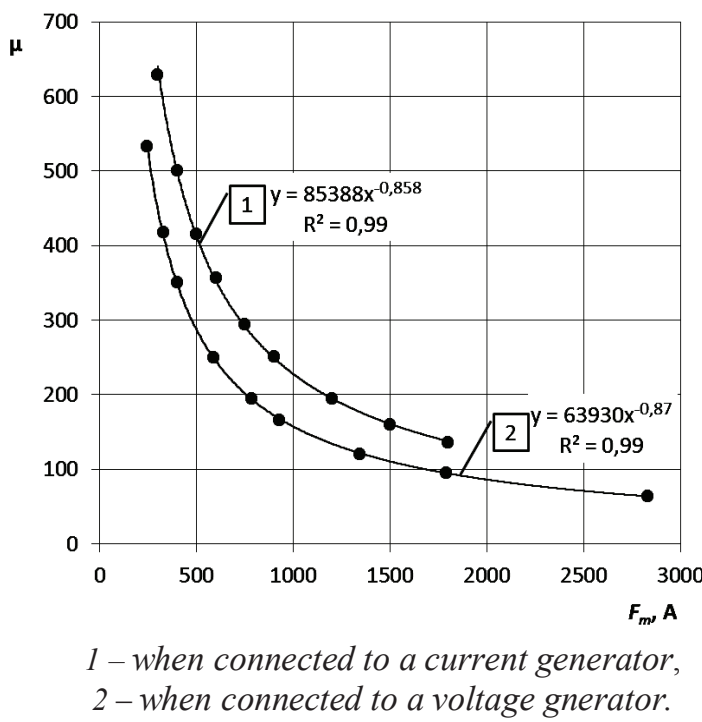

Fig. 4. Magnetic permeability dependence on the inductor MMF

The differences in curves 1 and 2 were caused by the transition to the linear description of electromagnetic processes: in fact, harmonic curves with nonlinear distortions characteristic of ferromagnetic materials were replaced with equivalent sinusoids. In this case the active power, which is released in the form of heat, is determined differently: the values of the power factor do not match. We note that curve $l$ is theoretical, since the current generators in induction heating of industrial equipment are not used, i.e. in practical calculations curve 2 must be used.

A similar approach to solving three-dimensional nonlinear equations of an electromagnetic field of ferromagnetic parallelepiped with a regular mesh of finite elements was described in [6]. Unlike the methods discussed, magnetic permeability is determined for each wall-adjacent block of finite elements by using the corresponding number of one-dimensional calculations. However, a three-dimensional model is used in the iterative process. On the one hand, such an approach can provide a high accuracy because magnetic permeability is not averaged over the volume of the ferromagnetic material; in fact, the magnetic permeability field is determined. On the other hand, a large amount of computation for solving real problems is inevitable.

The proposed method seems to be a compromise: it provides acceptable accuracy of the solution, it does not require a large amount of computation, and it is easy to implement.

\section{A method for calculating the inductors power}

The proposed method of finding the equivalent magnetic permeability can be used in calculation of the inductors power. The studies of the impact of different factors on 
magnetic permeability show that any three-dimensional inductor can be accurately approximated by a twodimensional model if the following two conditions are satisfied:

1) the inductor must be placed entirely within the heated body;

2) parts of the one or more inductors with multidirectional currents must be positioned from each other at a distance of at least 4-fold depth $\delta$ of the electromagnetic wave penetration in the body under consideration.

The value of $\delta$ is calculated by the formula:

$$
\delta=\sqrt{\frac{T}{\pi \gamma \mu}} .
$$

The wave penetration depth $\delta$ is arbitrary: it is the distance from the surface of the material with linear properties at which the magnetic field strength is attenuated by $e$ times. The generated real power is proportional to the square of the magnetic field. Therefore, in a layer with a thickness of $\delta$ about $86.5 \%$ of the total energy is released from the surface.

If two of these conditions are satisfied, an axisymmetric two-dimensional analog of the original inductor is constructed. The principle of the transition to the two-dimensional model is based on the equality of the lengths of axial lines of the original inductor and the axisymmetric inductor, i.e., the radius of the latter is calculated by the formula:

$$
R_{2 D}=\frac{L_{3 D}}{2 \pi},
$$

where $L_{3 D}$ is the length of a three-dimensional inductor along the axial line, $\mathrm{m}$.

Then, a two-dimensional axisymmetric model similar to that shown in Fig. 2 is constructed. In this case, a cross-sectional shape of the inductor is not modified.

The next step is the solution of the equation (5) and calculation of real power by the formula (7).

When calculating the power, this method allows taking into account non-linear characteristics of ferromagnetic materials and perform calculations of high-frequency inductors. For example, in accordance with (10) at $50 \mathrm{kHz}$ the final dimensions of elements in the steel material should not exceed $0.04 \mathrm{~mm}$ near the magnetic field sources. In a three-dimensional formulation, a similar calculation can be done only by supercomputers.

The proposed method is also effective for a simplified calculation of temperature fields of induction heating devices, assuming a uniform heat generation in the volume of the slots for the inductors [7].

To test the proposed method, we used data of physical experiment on heating the induction plate sized $500 \times 410 \mathrm{~mm}$ with four identical inductors used in vulcanization presses (for details, see [8]).

During the experiment, we recorded the power consumption readings for the plate, and its temperature at a place of control thermocouple location. The experimental results and calculated values of real power depending on the plate temperature are shown in Fig. 5. The average deviation of the calculated values from experimental data was $2.7 \%$.

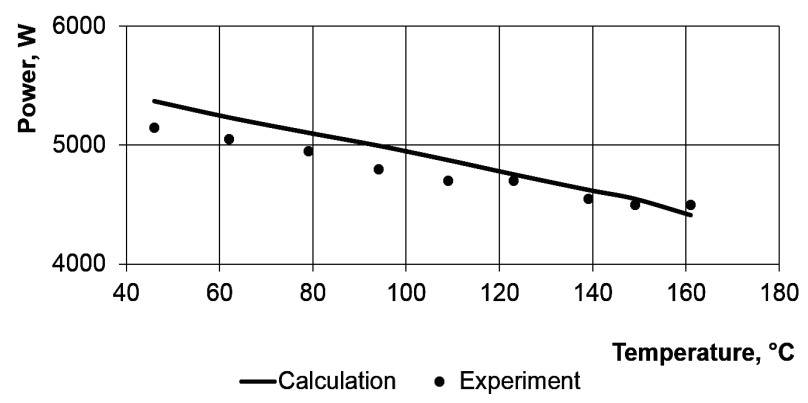

Fig. 5. Experimental data and calculated values compared

Since the calculation of the inductors power was made with application of the proposed method for determining the equivalent permeability, these results indirectly verify the accuracy of the magnetic permeability calculations.

\section{Results and discussion}

We described a method of determining the equivalent magnetic permeability of ferromagnetic materials. The proposed method is based on simplifying induction heating mathematical model equations to quasistationary linear equations in the complex representation. This simplification allows reducing the amount of computation by more than an order of magnitude.

Given the proposed method, we developed a method of calculating the inductors power, which can be effectively used in design of new inductors (including high-frequency inductors), as well as in a simplified calculation of temperature fields of induction heating devices.

This work was financially supported by Ministry of Education and Science of the Russian Federation within the basic part (project 8.7082.2017/8.9).

\section{References}

1. O. Bíró, K. Preis, IEEE Transactions on Magnetics. 25(4), 3145-3159 (1989)

2. A.O. Glebov, S.V. Karpov, S.V. Karpushkin. Autom. Remote Control, 75(6), 1120-1129 (2014)

3. S.M. Fu, Z.Y. Wu, S.Q. Cao, Advanced Materials Research, 753, 1035-1039 (2013)

4. J.L. Coulomb, J.C. Sabonnadière CAO en électrotechnique (Hermes, 1985)

5. N. Takahashi, T. Nakata, H. Morishige, COMPEL, 14(2/3), 57-75 (1995)

6. A. Canova, F. Dughiero, F. Fasolo, M. Forzan, F. Freschi, L. Giaccone, M. Repetto, IEEE Transactions on Magnetics, 45(3), 1855-1858 (2009) 
7. A.V. Ostroukh, A.O. Glebov, S.V. Karpov, S.V. Karpushkin, M.N. Krasnyanskiy. Am. J. Appl. Sci., 11(6), 939-946 (2014)

8. S.V. Karpushkin, S.V. Karpov, A.O. Glebov.

Transactions Tambov STU, 17(1), 110-120 (2011) 